\title{
Robust Stabilization of a Centrifugal Compressor With Spool Dynamics
}

\author{
Der-Cherng Liaw, Chau-Chung Song, and Jeng-Tze Huang
}

\begin{abstract}
Issues of global stabilization of a centrifugal compressor with spool dynamics are presented. Control schemes are designed for systems with and without system uncertainty. While activating only the close-coupled valve or the throttle as the single control input, backstepping tools are first used to achieve global stability of the working equilibrium for systems without uncertainty. The uncertainties in the spool dynamics and the compressor characteristic are known to be likely to happen in practical applications. Under such circumstances, the compressor torque and the close-coupled valve are then employed to achieve robust global stability of the working equilibrium via the Lyapunov redesign method. Numerical simulations are given to demonstrate the main results.
\end{abstract}

Index Terms-Backstepping, centrifugal compressor, global stabilization, Lyapunov method, spool dynamics.

\section{INTRODUCTION}

I T IS KNOWN that a centrifugal compressor achieves its optimal performance while working at the peak of the compressor map. However, the system might encounter surge if it is throttled beyond that critical point. Surge is known to be a one-dimensional oscillation involving the pressure rise and the mass flow which sharply reduces the compressor efficiency and can damage the compressor [1]. Therefore, the avoidance of the surge oscillation becomes an important issue for high-efficiency operation of centrifugal compression systems.

Recently, the control of surge behavior has attracted considerable attention (see the review in [2]) and particular interest has focused on global control and robustness issues [3]-[9] and [16]. For instance, Simon and Valavani [6] first proposed a sliding mode control design by using the close-coupled valve to robustly stabilize a compression system subject to the uncertainty in the compressor map. Gravdahl and Egeland [7] proposed a Lyapunov-based backstepping design to tackle the uncertainties in both compressor and throttle maps. However, these two papers did not consider the effect of spool dynamics whose transients might cause instabilities. Gravdahl and Egeland also proposed a control design to achieve robust exponential stability of the equilibrium by activating the close-coupled valve and/or the compressor torque [8], [18].

Manuscript received December 27, 2001; revised September 26, 2003 Manuscript received in final form March 19, 2004. Recommended by Associate Editor A. Banaszuk. This work was supported by the National Science Council, Taiwan, R.O.C. under Grants NSC-90-2213-E-009-102, NSC-912213-E-009-034, NSC-92-2213-E-009-014, and NSC-91-2212-E-216-019.

D.-C. Liaw is with the Department of Electrical and Control Engineering, National Chiao Tung University, Hsinchu 300, Taiwan, R.O.C. (e-mail: dcliaw@cc.nctu.edu.tw).

C.-C. Song is with the Department of Electrical Engineering, Chung Hua University, Hsinchu 300, Taiwan, R.O.C.

J.-T. Huang is with the Department of Electronics Engineering, Vanung University, Chungli 320, Taiwan, R.O.C.

Digital Object Identifier 10.1109/TCST.2004.833611
That result is based on a model with a specified nominal compressor map. Fink, Cumpsty, and Greitzer derived a three-state lumped parameter model for a centrifugal compression system that includes spool dynamics [17]. It was further detailed in [5] and [8]. Based on this model, control designs were also proposed to achieve global stability of the equilibrium without considering robustness issues [3], [4]. Though the spool dynamics might be very slow with respect to surge dynamics, the compressor characteristic is known (e.g., [3]-[5], [8], [17]) to be a function of spool speed. The robust control designs for centrifugal compression systems with the influence of spool dynamics remains an important issue.

In this brief, we propose control designs for centrifugal compression systems with and without system uncertainties. There are two main goals of this study. One is to study the global stabilization of general centrifugal compression systems without considering system uncertainties. Instead of using the full control efforts for guaranteeing system stabilization as those in [3]-[5], the Lyapunov function approach via single control input is proposed in this brief to tackle global system stabilization problem. The other is to achieve robust global stability for compressors with bounded matched-type uncertainty. This will be achieved by tuning both driving torque in spool dynamics and closed-couple valve only.

The brief is organized as follows. In Section II, the three-state lumped-parameter model for centrifugal compression systems is recalled. A brief description of compressor dynamics is also given to highlight the motivation of the study. It is followed by the application of backstepping approach to the global stabilization design for compression systems. The example compressor model presented in [3] is then adopted in Section VI for numerical study to demonstrate the applications of the proposed designs. Finally, the conclusion is given in Section V to summarize the main results.

\section{DyNAMIC EQUATIONS FOR A CENTRIFUGAL COMPRESSOR}

A basic centrifugal compression system is consisted of inlet duct, compressor, outlet duct, plenum, exit duct, and a control throttle (see, e.g., [5, Fig. 2]). Here, the plenum is a simplified model of the combustion chamber and the throttle can be regarded as a simplified model of a turbine. In general, the fluid dynamics of a compression system can only be fully described by a set of partial differential equations. To facilitate the analysis, lumped-parameter model of compressors have been recently derived (e.g., [10]). Assume that the flow in the compression system is one-dimensional and incompressible, and the plenum dimension is large enough so that the pressure in the plenum is spatially uniform. In addition, the thermodynamic process of the system is assumed to be isentropic. Based on 
these assumptions, a lumped-parameter three-state model (e.g., [3]-[5], [17]) is recalled below

$$
\begin{aligned}
\frac{d P_{p}}{d t} & =\frac{a^{2}}{V_{p}}\left(\dot{m}_{c}-\dot{m}_{t}\right) \\
\frac{L}{A} \frac{d \dot{m}_{c}}{d t} & =P_{c}-P_{p} \\
J \frac{d \omega}{d t} & =\tau_{t}-\tau_{c}
\end{aligned}
$$

where $P_{p}, \dot{m}_{c}$, and $\omega$ denote the pressure in the plenum, compressor mass flow rate, and spool speed, respectively. The definitions of remaining parameters and variables can be referred to (e.g., [3], [4], [8], [17]). Note that (1) and (2) are equivalent to the model introduced by Greitzer [10]. In (3), the angular speed of the compressor $\omega$ is included as one of the system states. Equation (1) describes the mass balance in the plenum, while (2) describes the momentum balance in the duct.

By suitably nondimensionalizing system (1)-(3) and assuming the throttle opening is a smooth function, the system equations can be rewritten as follows [3]:

$$
\begin{aligned}
\dot{\tilde{\omega}} & =\bar{a}\left\{\tau_{t}-\Gamma(\phi, \tilde{\omega})\right\} \\
\dot{\phi} & =\bar{b}\left\{\psi_{c}(\phi, \tilde{\omega})-\psi\right\} \\
\dot{\psi} & =\bar{c}\{\phi-F(\gamma, \psi)\} .
\end{aligned}
$$

Here, $\psi_{c}$ denotes the compressor characteristic map which is a function of both mass flow and spool speed. Note that, instead of adopting $\sigma \phi \tilde{\omega}$ and $\gamma \sqrt{\psi}$ for the approximate compressor torque and throttle function as in [3], here, we use $\Gamma(\phi, \tilde{\omega})$ and $F(\gamma, \psi)$ to denote the general compressor torque function and throttle pressure map, respectively. In addition, $\gamma$ is proportional to the throttle opening, while $\bar{a}>0, \bar{b}>0$ and $\bar{c}>0$. The physical interpretation of the remaining parameters in (4)-(6) can be found in, e.g., [3], [4], [8], and [17].

\section{Control Designs}

In this section, Lyapunov-function-based global control schemes for the centrifugal compressor model (4)-(6), without considering system uncertainty, are first proposed to guarantee system stability by using either the close-coupled valve or the throttle opening as the unique actuator. This derivation is followed by a robust design that compensates for system uncertainties.

It is clear that the equilibrium of (4)-(6) with a constant driving torque $\tau_{t}=\tau_{0}$ can be easily determined by equating the right-hand side of (4)-(6) to zero. Denote $x^{0}=\left(\tilde{\omega}^{0}, \phi^{0}, \psi^{0}\right)^{T}$ such an equilibrium. We then have

$$
\begin{aligned}
\tau_{0} & =\Gamma\left(\phi^{0}, \tilde{\omega}^{0}\right) \\
\phi^{0} & =F\left(\gamma^{0}, \psi^{0}\right) \\
\psi^{0} & =\psi_{c}\left(\phi^{0}, \tilde{\omega}^{0}\right) .
\end{aligned}
$$

Note that the system equilibrium points can hence be obtained from the intersection of throttle and compressor map for given $\tilde{\omega}^{0}$ and $\gamma^{0}$.

Next, we study the local stability around the equilibrium point $x^{0}$. Let $x=\left(x_{1}, x_{2}, x_{3}\right)^{T}$ with $x_{1} \triangleq \tilde{\omega}-\tilde{\omega}^{0}, x_{2} \triangleq \phi-\phi^{0}$ and $x_{3} \triangleq \psi-\psi^{0}$. For a general centrifugal compressor, we might have control input signals from the compressor driving torque, the close-coupled valve and the throttle opening. Let $u_{1}=\tau_{t}-$ $\tau_{0}, u_{2}$ and $u_{3}=\gamma-\gamma^{0}$ be the extra applied compressor torque, the close-coupled valve control, and the throttle opening control, respectively. The system of (4)-(6) can then be rewritten as

$$
\begin{aligned}
& \dot{x}_{1}=\bar{a}\left\{f\left(x_{1}, x_{2}\right)+u_{1}\right\} \\
& \dot{x}_{2}=\bar{b}\left\{C_{s s}\left(x_{1}, x_{2}\right)-x_{3}+u_{2}\right\} \\
& \dot{x}_{3}=\bar{c}\left\{x_{2}+F\left(\gamma^{0}, \psi^{0}\right)-F\left(u_{3}+\gamma^{0}, x_{3}+\psi^{0}\right)\right\}
\end{aligned}
$$

where

$$
C_{s s}\left(x_{1}, x_{2}\right) \triangleq \psi_{c}\left(x_{2}+\phi^{0}, x_{1}+\tilde{\omega}^{0}\right)-\psi_{c}\left(\phi^{0}, \tilde{\omega}^{0}\right)
$$

and

$$
f\left(x_{1}, x_{2}\right) \triangleq \Gamma\left(\phi^{0}, \tilde{\omega}^{0}\right)-\Gamma\left(x_{2}+\phi^{0}, x_{1}+\tilde{\omega}^{0}\right) .
$$

For a given $\gamma^{0}$, the linearization at the equilibrium point $x^{0}$ for the uncontrolled model of system (10)-(12) gives

$$
\dot{x}=A x
$$

where

$$
A=\left(\begin{array}{ccc}
-\bar{a} \frac{\partial f}{\partial \tilde{\omega}}(0,0) & -\bar{a} \frac{\partial f}{\partial \phi}(0,0) & 0 \\
\bar{b} \frac{\partial C_{s s}}{\partial \tilde{\omega}}(0,0) & \bar{b} \frac{\partial C_{s s}}{\partial \phi}(0,0) & -\bar{b} \\
0 & \bar{c} & -\bar{c} \frac{\partial F}{\partial \psi}(0,0)
\end{array}\right) .
$$
have

By solving the characteristic polynomial of system (15), we

$$
\operatorname{det}\left(\lambda I_{3 \times 3}-A\right)=\lambda^{3}+c_{1} \lambda^{2}+c_{2} \lambda+c_{3}
$$

where

$$
\begin{aligned}
c_{1}= & \bar{a} \frac{\partial \Gamma}{\partial \tilde{\omega}}-\bar{b} \frac{\partial \psi_{c}}{\partial \phi}+\bar{c} \frac{\partial F}{\partial \psi} \\
c_{2}= & \bar{b} \bar{c}+\bar{a} \bar{b} \frac{\partial \psi_{c}}{\partial \tilde{\omega}} \frac{\partial \Gamma}{\partial \phi}-\bar{a} \bar{b} \frac{\partial \Gamma}{\partial \tilde{\omega}} \frac{\partial \psi_{c}}{\partial \phi}-\bar{b} \bar{c} \frac{\partial \psi_{c}}{\partial \phi} \frac{\partial F}{\partial \psi} \\
& +\bar{a} \bar{c} \frac{\partial \Gamma}{\partial \tilde{\omega}} \frac{\partial F}{\partial \psi} \\
c_{3}= & \bar{a} \bar{b} \bar{c}\left(\frac{\partial \Gamma}{\partial \tilde{\omega}}+\frac{\partial \psi_{c}}{\partial \tilde{\omega}} \frac{\partial \Gamma}{\partial \phi} \frac{\partial F}{\partial \psi}-\frac{\partial \Gamma}{\partial \tilde{\omega}} \frac{\partial \psi_{c}}{\partial \phi} \frac{\partial F}{\partial \psi}\right) .
\end{aligned}
$$

Applying the Routh-Hurwitz criterion to system (15), we then have the next stability result.

Lemma 1: The equilibrium point $x^{0}$ for the uncontrolled version of system (10)-(12) is asymptotically stable if $c_{1}>0$, $c_{3}>0$ and $c_{1} c_{2}>c_{3}$, where $c_{i}$ are defined in (18)-(20).

From [5] and [17], the compressor torque is known to be strictly increasing with respect to $\phi$ only for $\phi>0$. For simplicity and without loss of generality, we make the following hypothesis to facilitate the stabilization design.

Hypothesis 1: The compressor pressure map $\psi_{c}$ is a monotonically increasing function with respect to the angular velocity of compressor. Moreover, the throttle function $F$ is also strictly increasing function with respect to the corresponding variables and the compressor torque $\Gamma$ is strictly increasing with respect to $\phi$ for $\phi>0$.

From Hypothesis 1 , we have $\partial \psi_{c} / \partial \tilde{\omega}>0, \partial F / \partial \psi>0$ and $\partial \Gamma / \partial \tilde{\omega}>0$. Moreover, we have $\partial \Gamma / \partial \phi>0$ for $\phi>0$. The next corollary follows readily from Lemma 1 . 
Corollary 1: Suppose Hypothesis 1 holds. Then an equilibrium point $x^{0}$ with $\phi^{0}>0$ for the uncontrolled version of system (10)-(12) is asymptotically stable if $\partial \psi_{c} / \partial \phi<0$.

Note that, the result of Corollary 1 agrees with that of [11] and highlights the local stability of system equilibrium with negative slope of $\psi_{c}$ versus the mass flow $\phi$. However, the domain of attraction for the uncontrolled equilibrium points decreases as the throttle closes [12]. In the following, we propose control laws to globally stabilize a given system equilibrium. For general applications, the pressure variable $\psi$ is known to be nonnegative. This will be in effect in the remaining of this brief. In Sections III-A and III-B, we will study the global stabilization problem for system (10)-(12) with explicit knowledge of system model. The robust design issue will be discussed in Section III-C.

\section{A. Close-Coupled Valve Control}

It is clear that system (10)-(12) is completely controllable when all three control inputs are available. In this brief, we study system stabilization with one or two control inputs. First, we consider the global stabilization design for system (10)-(12) with the close-coupled valve $u_{2}$ being the unique available control.

Motivated by the polynomial function of compressor torque as that in [3] and [4], we assume the function $f\left(x_{1}, x_{2}\right)$ is smooth and can be decomposed as

$$
f\left(x_{1}, x_{2}\right)=f\left(x_{1}, 0\right)+x_{2} \tilde{f}\left(x_{1}, x_{2}\right) .
$$

Clearly, $f\left(x_{1}, 0\right)$ is the so-called "zero dynamics" of the subsystem (10) with $u_{1}=0$. Since $\Gamma$ is a strictly increasing function with respect to $\tilde{\omega}$, it is clear from (14) that $x_{1} f\left(x_{1}, 0\right)<0$ for $x_{1} \neq 0$.

Let

$$
V(x)=\frac{1}{2}\left(\frac{1}{\bar{a}} x_{1}^{2}+\frac{1}{\bar{b}} x_{2}^{2}+\frac{1}{\bar{c}} x_{3}^{2}\right)
$$

be an energy-like Lyapunov function candidate for system (10)-(12). Taking the time derivative of $V(x)$ along trajectories of system (10)-(12) for $u_{1}=u_{3}=0$, we have

$$
\begin{aligned}
\dot{V}(x)= & \frac{1}{\bar{a}} x_{1} \dot{x}_{1}+\frac{1}{\bar{b}} x_{2} \dot{x}_{2}+\frac{1}{\bar{c}} x_{3} \dot{x}_{3} \\
= & x_{1} f\left(x_{1}, 0\right)+x_{1} x_{2} \tilde{f}\left(x_{1}, x_{2}\right)+x_{2} C_{s s}\left(x_{1}, x_{2}\right) \\
& +x_{3}\left\{F\left(\gamma^{0}, \psi^{0}\right)-F\left(\gamma^{0}, x_{3}+\psi^{0}\right)\right\}+x_{2} u_{2} .
\end{aligned}
$$

Suppose $F$ is a strictly increasing function with respect to $\psi$. It is clear that $x_{3}\left\{F\left(\gamma^{0}, \psi^{0}\right)-F\left(\gamma^{0}, x_{3}+\psi^{0}\right)\right\} \leq 0$ for all $x_{3}$ and the equality holds only at $x_{3}=0$. Thus, $V(x)$ in (23) will be a negative definite function if the applied control input $u_{2}$ is chosen so that $V_{1}$ defined in (24) is a negative definite function of $x_{2}$ only

$$
V_{1}:=x_{1} x_{2} \tilde{f}\left(x_{1}, x_{2}\right)+x_{2} C_{s s}\left(x_{1}, x_{2}\right)+x_{2} u_{2} .
$$

Obviously, one of such choices is

$$
u_{2}=-x_{1} \tilde{f}\left(x_{1}, x_{2}\right)-C_{s s}\left(x_{1}, x_{2}\right)-u\left(x_{2}\right)
$$

where $u\left(x_{2}\right) x_{2}$ is a positive definite function for all $x_{2} \in R$. We then have $\dot{V}(x) \leq 0$ for all $x \in R^{3}$ and $\dot{V}(x)=0$ only occurs at $x=0$. Note that, similar designs have been given in [7], [8] to dominate the effect of $x_{2} C_{s s}\left(x_{1}, x_{2}\right)$ for making $\dot{V}(x) \leq 0$. However, the proposed design in (25) provides more freedom in the selection of controller.

Employing Lyapunov stability criteria, we have the next global stabilization result following directly from the discussions above.

Theorem 1: Suppose Hypothesis 1 holds. The system equilibrium $\left(\tilde{\omega}^{0}, \phi^{0}, \psi^{0}\right)$ of system (10)-(12) can then be globally stabilized by the close-coupled valve control. One of control laws is given in (25).

Remark 1: The proposed control design given in Theorem 1 calls for the exact knowledge of the functions $C_{s s}\left(x_{1}, x_{2}\right)$ and $f\left(x_{1}, x_{2}\right)$ (as in (13)-(14)). However, it requires no activation of the compressor torque to stabilize the spool dynamics. This is the main advantage of the above design.

\section{B. Throttle Control}

Next, we study the case of which the throttle setting is the unique applied control force. Though the throttle control is known to be easier for most practical implementations, however, the uncertainty of a compression system can not be directly cancelled by throttle control alone. Motivated by Krstić et al. [13] and Liaw et al. [16], we use the backstepping control approach to construct a practically global stabilization scheme for system (10)-(12).

It is observed from (10)-(12) that the throttle-controlled version of system (10)-(12) becomes

$$
\begin{aligned}
& \dot{x}_{1}=\bar{a}\left\{f\left(x_{1}, 0\right)+x_{2} \tilde{f}\left(x_{1}, x_{2}\right)\right\} \\
& \dot{x}_{2}=\bar{b}\left\{C_{s s}\left(x_{1}, x_{2}\right)-x_{3}\right\} \\
& \dot{x}_{3}=\bar{c}\left\{x_{2}+F\left(\gamma^{0}, \psi^{0}\right)-F\left(u_{3}+\gamma^{0}, x_{3}+\psi^{0}\right)\right\} .
\end{aligned}
$$

Following the backstepping design procedure [14], we first try to globally stabilize the subsystem (26)-(27) by treating $x_{3}$ as a virtual control input. According to the discussions in Section III-A, $x_{3}$ can be chosen such that for any $x_{1} \in R, V_{2}$ as defined in (29) below is a negative definite function of $x_{2}$

$$
V_{2}:=x_{1} x_{2} \tilde{f}\left(x_{1}, x_{2}\right)+x_{2} C_{s s}\left(x_{1}, x_{2}\right)-x_{2} x_{3} \text {. }
$$

Let $x_{3 d}$ be such a designed virtual control $x_{3}$ which makes $V_{2}$ be a negative definite function of $x_{2}$. It is obvious that one of such choices is

$$
x_{3 d}=x_{1} \tilde{f}\left(x_{1}, x_{2}\right)+C_{s s}\left(x_{1}, x_{2}\right)+x_{2} .
$$

Next, denote the error term $\xi:=x_{3}-x_{3 d}$. The throttle control system (26)-(28) can then be rewritten as

$$
\begin{aligned}
\dot{x}_{1}= & \bar{a}\left\{f\left(x_{1}, 0\right)+x_{2} \tilde{f}\left(x_{1}, x_{2}\right)\right\} \\
\dot{x}_{2}= & \bar{b}\left\{C_{s s}\left(x_{1}, x_{2}\right)-x_{3 d}\right\}-\bar{b} \xi \\
\dot{\xi}= & \bar{c}\left\{x_{2}+F\left(\gamma^{0}, \psi^{0}\right)\right\} \\
& -\bar{c} F\left(u_{3}+\gamma^{0}, \xi+x_{3 d}+\psi^{0}\right)-\dot{x}_{3 d} .
\end{aligned}
$$


Choose a Lyapunov function candidate $V\left(x_{1}, x_{2}, \xi\right)$ for (31)-(33) as

$$
V\left(x_{1}, x_{2}, \xi\right)=\frac{1}{2}\left(\frac{1}{\bar{a}} x_{1}^{2}+\frac{1}{\bar{b}} x_{2}^{2}+\frac{1}{\bar{c}} \xi^{2}\right) .
$$

The time derivative of $V$ along the trajectories of system (31)-(33) can then be calculated as

$$
\begin{aligned}
\dot{V}\left(x_{1}, x_{2}, \xi\right)= & \frac{1}{\bar{a}} x_{1} \dot{x}_{1}+\frac{1}{\bar{b}} x_{2} \dot{x}_{2}+\frac{1}{\bar{c}} \xi \dot{\xi} \\
= & x_{1} f\left(x_{1}, 0\right)-x_{2}^{2}+\xi\left\{F\left(\gamma^{0}, \psi^{0}\right)\right. \\
& \left.-F\left(u_{3}+\gamma^{0}, \xi+x_{3 d}+\psi^{0}\right)-\frac{1}{\bar{c}} \dot{x}_{3 d}\right\} .
\end{aligned}
$$

It is observed from (35) that $\dot{V}\left(x_{1}, x_{2}, \xi\right)$ will be a negative definite function for $\left(x_{1}, x_{2}, \xi\right)^{T} \in R^{3}$ if the applied throttle control $u_{3}$ is chosen to make

$$
V_{3}:=\xi\left\{F\left(\gamma^{0}, \psi^{0}\right)-F\left(u_{3}+\gamma^{0}, \xi+x_{3 d}+\psi^{0}\right)-\frac{1}{\bar{c}} \dot{x}_{3 d}\right\}
$$

a negative definite function with respect to $\xi$.

According to Lyapunov stability criteria, we then have the next theorem.

Theorem 2: Suppose Hypothesis 1 holds. The equilibrium point $\left(\tilde{\omega}^{0}, \phi^{0}, \psi^{0}\right)$ of system (31)-(33) is globally stabilized by the backstepping throttle control laws $\gamma=\gamma^{0}+u_{3}$ where $u_{3}$ makes $V_{3}$ in (36) a negative definite function with respect to $\xi$.

Note that, it is clear from Theorem 2 and (36) that the proposed control law depends on the existence of $u_{3}$. To demonstrate such an application, consider the throttle function adopted from [3] and [11] as given by $F(\gamma, \psi)=\gamma \sqrt{\psi}$. In most practical applications, the total pressure rise $\psi=\xi+x_{3 d}+\psi^{0}$ is a positive value. From (36), one of choices for $u_{3}$ is that

$$
\begin{aligned}
u_{3}= & \frac{1}{\sqrt{\xi+x_{3 d}+\psi^{0}}} \\
& \times\left\{\gamma^{0}\left(\sqrt{\psi^{0}}-\sqrt{\xi+x_{3 d}+\psi^{0}}\right)-\frac{1}{\bar{c}} \dot{x}_{3 d}+\xi\right\}
\end{aligned}
$$

provided with $\xi+x_{3 d}+\psi^{0} \neq 0$. It is clear from (37) that the throttle control input $u_{3}$ as in (36) for the design of Theorem 2 is solvable.

\section{Robust Control Designs}

Now, we consider the robust design for centrifugal compressors with uncertainties appearing in the compressor characteristic $C_{s s}\left(x_{1}, x_{2}\right)$ and the compressor torque $f\left(x_{1}, x_{2}\right)$. The proposed scheme is consisted of two loops. The inner loop is intended to provide global stabilization of the nominal model, while the outer control loop will cancel the effect of system uncertainties. Details are given as follows.

Let the compressor torque and compressor characteristic be

$$
\begin{aligned}
f\left(x_{1}, x_{2}\right) & =\bar{f}\left(x_{1}, x_{2}\right)+\Delta f\left(x_{1}, x_{2}\right) \\
C_{s s}\left(x_{1}, x_{2}\right) & =\bar{C}_{s s}\left(x_{1}, x_{2}\right)+\Delta C_{s s}\left(x_{1}, x_{2}\right)
\end{aligned}
$$

where $\bar{f}\left(x_{1}, x_{2}\right)$ and $\bar{C}_{s s}\left(x_{1}, x_{2}\right)$ denote nominal models, while $\Delta f\left(x_{1}, x_{2}\right)$ and $\Delta C_{s s}\left(x_{1}, x_{2}\right)$ are uncertainties for $f\left(x_{1}, x_{2}\right)$ and $C_{s s}\left(x_{1}, x_{2}\right)$, respectively.

Assume that

$$
\begin{array}{r}
\left|\Delta f\left(x_{1}, x_{2}\right)\right| \leq \delta_{1}\left(x_{1}, x_{2}\right) \\
\left|\Delta C_{s s}\left(x_{1}, x_{2}\right)\right| \leq \delta_{2}\left(x_{1}, x_{2}\right)
\end{array}
$$

where $\delta_{1}\left(x_{1}, x_{2}\right)$ and $\delta_{2}\left(x_{1}, x_{2}\right)$ are two known bounded and positive definite functions.

In this study, we assume the spool driving torque $u_{1}$ and closed-couple valve $u_{2}$ are two available control inputs, which makes the control system of (10)-(12) with $u_{3}=0$ satisfy the so-called "matching conditions."

Let $u_{1}=\Delta u_{1}$ and $u_{2}=\bar{u}_{2}+\Delta u_{2}$, where $\bar{u}_{2}$ is for the inner control loop design. The two extra inputs $\Delta u_{1}$ and $\Delta u_{2}$ are for the outer control loop, which will be designed to compensate the dynamic effect by system uncertainty. System (10)-(12) can then be rewritten as

$$
\begin{aligned}
& \dot{x}_{1}=\bar{a}\left\{\bar{f}\left(x_{1}, x_{2}\right)+\Delta f\left(x_{1}, x_{2}\right)+\Delta u_{1}\right\} \\
& \dot{x}_{2}=\bar{b}\left\{\bar{C}_{s s}\left(x_{1}, x_{2}\right)+\Delta C_{s s}\left(x_{1}, x_{2}\right)-x_{3}+\bar{u}_{2}+\Delta u_{2}\right\} \\
& \dot{x}_{3}=\bar{c}\left\{x_{2}+F\left(\gamma^{0}, \psi^{0}\right)-F\left(\gamma^{0}, x_{3}+\psi^{0}\right)\right\}
\end{aligned}
$$

As motivated by the results of Section III-A, we choose $V(x)$ given in (22) as a Lyapunov function candidate for the system (10)-(12). Taking the time derivative of $V(x)$ along the trajectories of system (41)-(43), we have

$$
\begin{aligned}
\dot{V}(x)= & \frac{1}{\bar{a}} x_{1} \dot{x}_{1}+\frac{1}{\bar{b}} x_{2} \dot{x}_{2}+\frac{1}{\bar{c}} x_{3} \dot{x}_{3} \\
= & x_{1} \bar{f}\left(x_{1}, x_{2}\right)+x_{3}\left\{F\left(\gamma^{0}, \psi^{0}\right)-F\left(\gamma^{0}, x_{3}+\psi^{0}\right)\right\} \\
& +x_{2} \bar{C}_{s s}\left(x_{1}, x_{2}\right)+x_{2} \bar{u}_{2}+x_{1} \Delta u_{1} \\
& +x_{1} \Delta f\left(x_{1}, x_{2}\right)+x_{2} \Delta u_{2}+x_{2} \Delta C_{s s}\left(x_{1}, x_{2}\right) .
\end{aligned}
$$

Following the design in Section III-A, we choose

$$
\bar{u}_{2}=-x_{1} \tilde{f}\left(x_{1}, x_{2}\right)-\bar{C}_{s s}\left(x_{1}, x_{2}\right)-x_{2}
$$

for the inner loop control. Note that, as mentioned in Section III-A, the term $x_{2}$ in (44) can be replaced by a function $u\left(x_{2}\right)$ with $x_{2} u\left(x_{2}\right)$ being a positive definite function. Such a design provides more freedom in the selection of controller than that in [7], [8]. We then have

$\dot{V}(x)=x_{1} f\left(x_{1}, 0\right)+x_{3}\left\{F\left(\gamma^{0}, \psi^{0}\right)-F\left(\gamma^{0}, x_{3}+\psi^{0}\right)\right\}$
$-x_{2}^{2}+x_{1}\left\{\Delta u_{1}+\Delta f\left(x_{1}, x_{2}\right)\right\}+x_{2}\left\{\Delta u_{2}+\Delta C_{s s}\left(x_{1}, x_{2}\right)\right\}$.

It is clear that $\dot{V}(x)$ in (45) will be a negative definite function if the applied control inputs $\Delta u_{1}$ and $\Delta u_{2}$ are chosen to make $x_{1}\left\{\Delta u_{1}+\Delta f\left(x_{1}, x_{2}\right)\right\}+x_{2}\left\{\Delta u_{2}+\Delta C_{s s}\left(x_{1}, x_{2}\right)\right\} \leq 0$. Obviously, one of such choices are

$$
\begin{aligned}
\Delta u_{1} & =-\operatorname{sign}\left(x_{1}\right) \cdot \delta_{1}\left(x_{1}, x_{2}\right) \\
\text { and } \Delta u_{2} & =-\operatorname{sign}\left(x_{2}\right) \cdot \delta_{2}\left(x_{1}, x_{2}\right) .
\end{aligned}
$$

This leads to the next result.

Theorem 3: Suppose Hypothesis 1 holds. The equilibrium points $\left(\tilde{\omega}^{0}, \phi^{0}, \psi^{0}\right)$ of system (41)-(43) can be globally stabilized by the control as given in (44) and (46)-(47) with respect to the uncertainties satisfying the conditions of (39)-(40). 

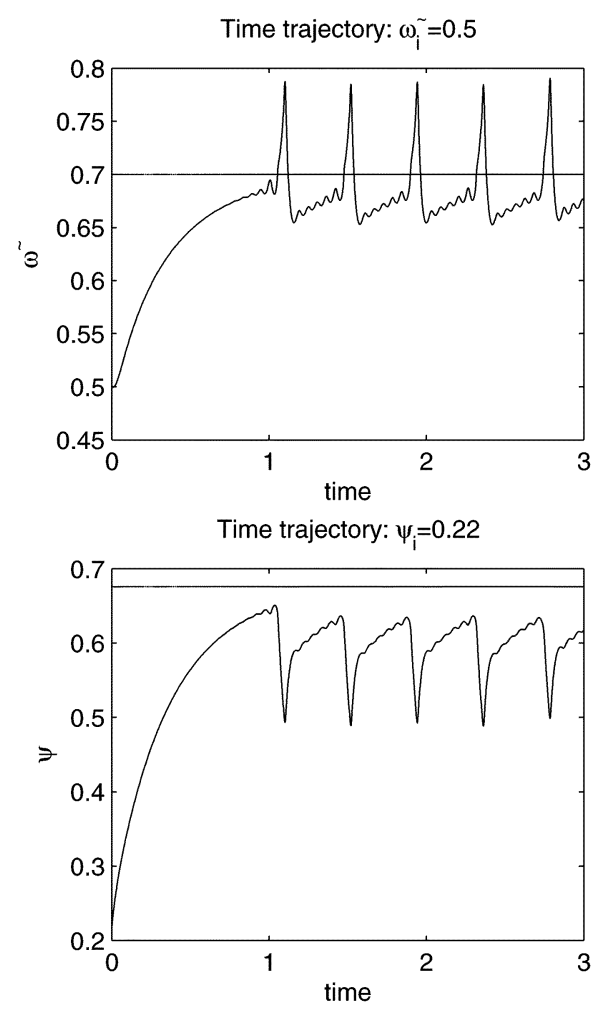

Fig. 1. Surge trajectories in open-loop dynamics.

Remark 2: Due to the sign effect, the above control law might introduce "chattering" behavior into the system dynamics. To avoid chattering behavior, a boundary layer around the equilibrium can be introduced within which a linear feedback control can operate. However, in general, only practical stability can be assured by such a continuous control design. Another concern for the practical implementation is the limitations of the actuators. As depicted in Fig. 2, the actuator limitations might contribute to the longer settling time while also providing system stabilization.

\section{NUMERICAL RESULTS}

In the following, numerical simulations for a example compression system will be given to demonstrate the effectiveness of the proposed control laws. Here, we adopt the model with same system parameters and numerical values as those in [3] for system performance comparison. As depicted in Fig. 1, the time responses of the uncontrolled model fall into a violent surge oscillation. These time responses are very similar to the ones given in [3]. In the following, the control algorithms developed in Section III are applied to system (10)-(12) to achieve global stability.

Case 1. Close-Coupled Valve Control: The control algorithm in (25) is first applied to system (10)-(12) with $\psi_{c}$ given in [3], which gives

$u_{2}=\sigma x_{1}\left(x_{1}+\tilde{\omega}^{0}\right)-\left\{\psi_{c}\left(x_{2}+\phi^{0}, x_{1}+\tilde{\omega}^{0}\right)-\psi_{c}\left(\phi^{0}, \tilde{\omega}^{0}\right)\right\}-x_{2}$.

As depicted in Fig. 2, the time responses of the control system are quickly brought back to the system equilibrium by control input $u_{2}$. Compared with the results presented in [3], it is clear

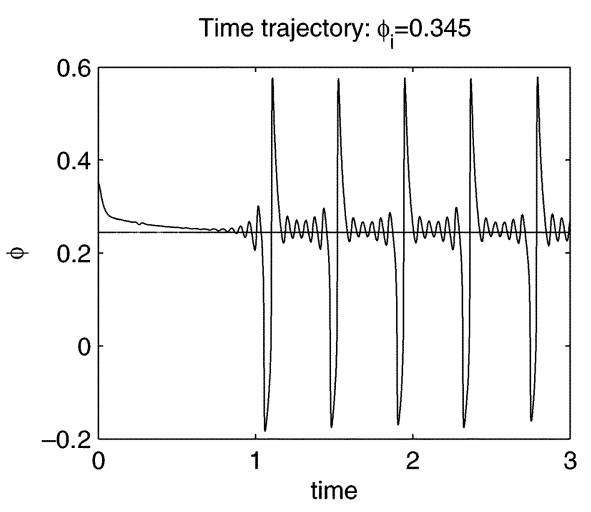

Phase plane: $x_{0}=\left[\begin{array}{lll}0.7 & 0.245 & 0.676\end{array}\right]$

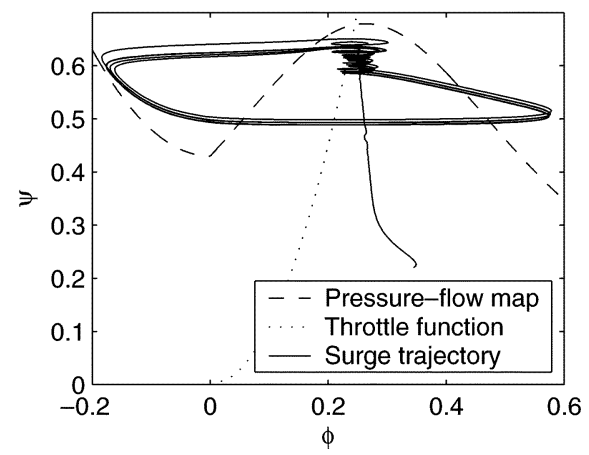

that two different designs can be applied to achieving global stabilization. However, both throttle and compressor torque are used in [3] to guarantee system stabilization, while we use only one closed-couple valve control for making system stable.

In order to check the effect of the actuator limitation on system performance, two different limit values of 0.1 and 0.2 have been set up for the $u_{2}$ in the numerical simulation. As depicted in Fig. 2, these actuator limitations will cause longer settling time while also providing system stabilization.

Case 2. Throttle Control: Following the design presented in Section III-B with $u_{3}$ as given in (37), we then have the modified model as given by

$$
\begin{aligned}
\dot{x}_{1} & =\bar{a} f\left(x_{1}, x_{2}\right) \\
\dot{x}_{2} & =\bar{b}\left\{C_{s s}\left(x_{1}, x_{2}\right)-x_{3}\right\} \\
\dot{\xi} & =\bar{c}\left(x_{2}-\xi\right)
\end{aligned}
$$

where $\xi=x_{3}-x_{3 d}$ with

$$
\begin{array}{r}
x_{3 d}=-\sigma x_{1}\left(x_{1}+\tilde{\omega}^{0}\right)+\left\{\psi_{c}\left(x_{2}+\phi^{0}, x_{1}+\tilde{\omega}^{0}\right)\right. \\
\left.-\psi_{c}\left(\phi^{0}, \tilde{\omega}^{0}\right)\right\}+x_{2} .
\end{array}
$$

The simulation results for the closed-loop system with the applied control effort are given in Fig. 3, which shows the effectiveness of system stabilization by the throttle control alone.

Case 3. Robust Control: Next, we present the results for robust design. The extra control inputs in (46)-(47) are applied to system (41)-(43) in order to compensate the uncertainties embedded in system dynamics. Motivated by [15], in this study the uncertainties are chosen to be

$$
\Delta f\left(x_{1}, x_{2}\right)=\Delta C_{s s}\left(x_{1}, x_{2}\right)=0.1 \sin \left(10 x_{2}\right) .
$$



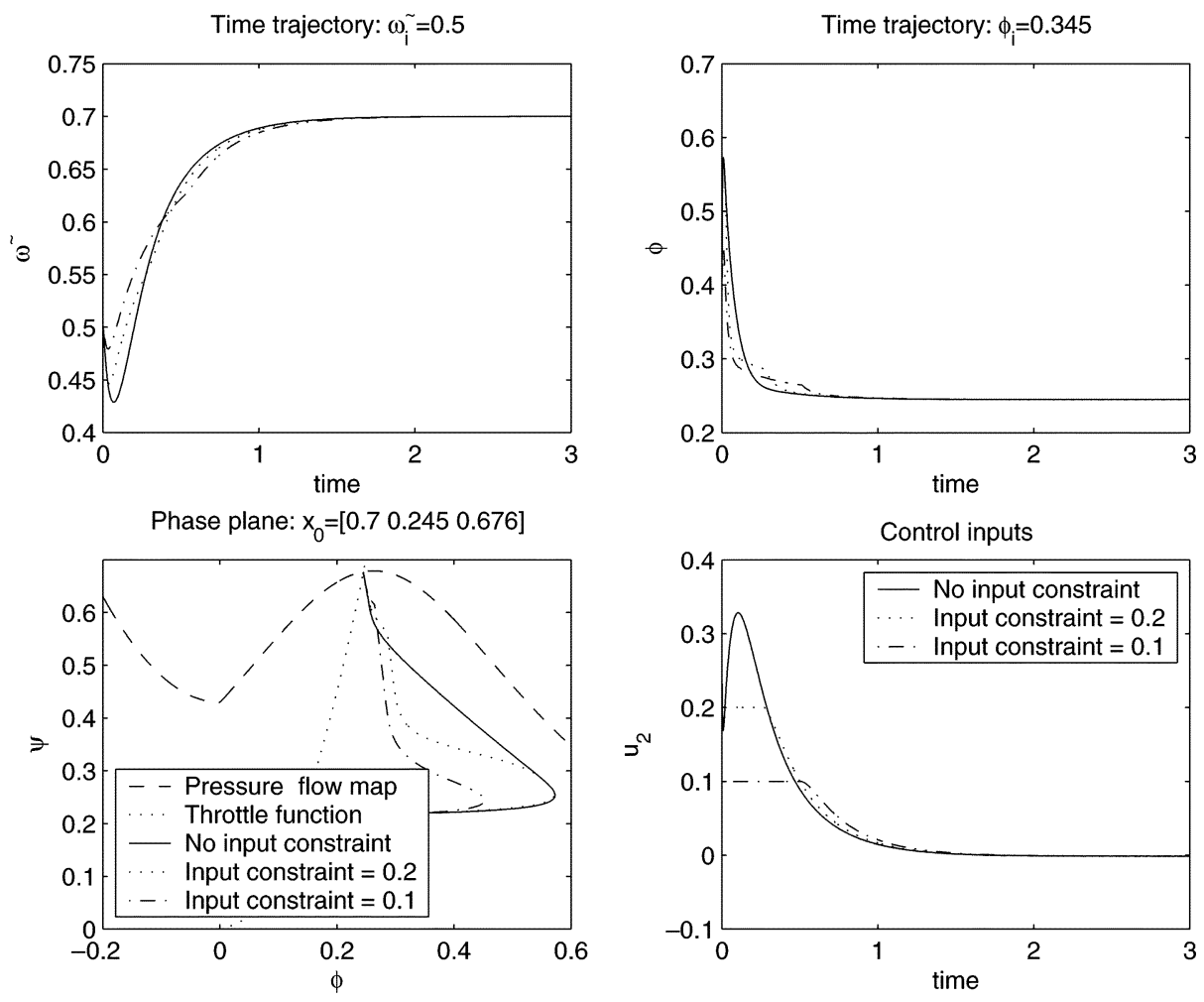

Fig. 2. Time trajectories for system with close-coupled valve control.
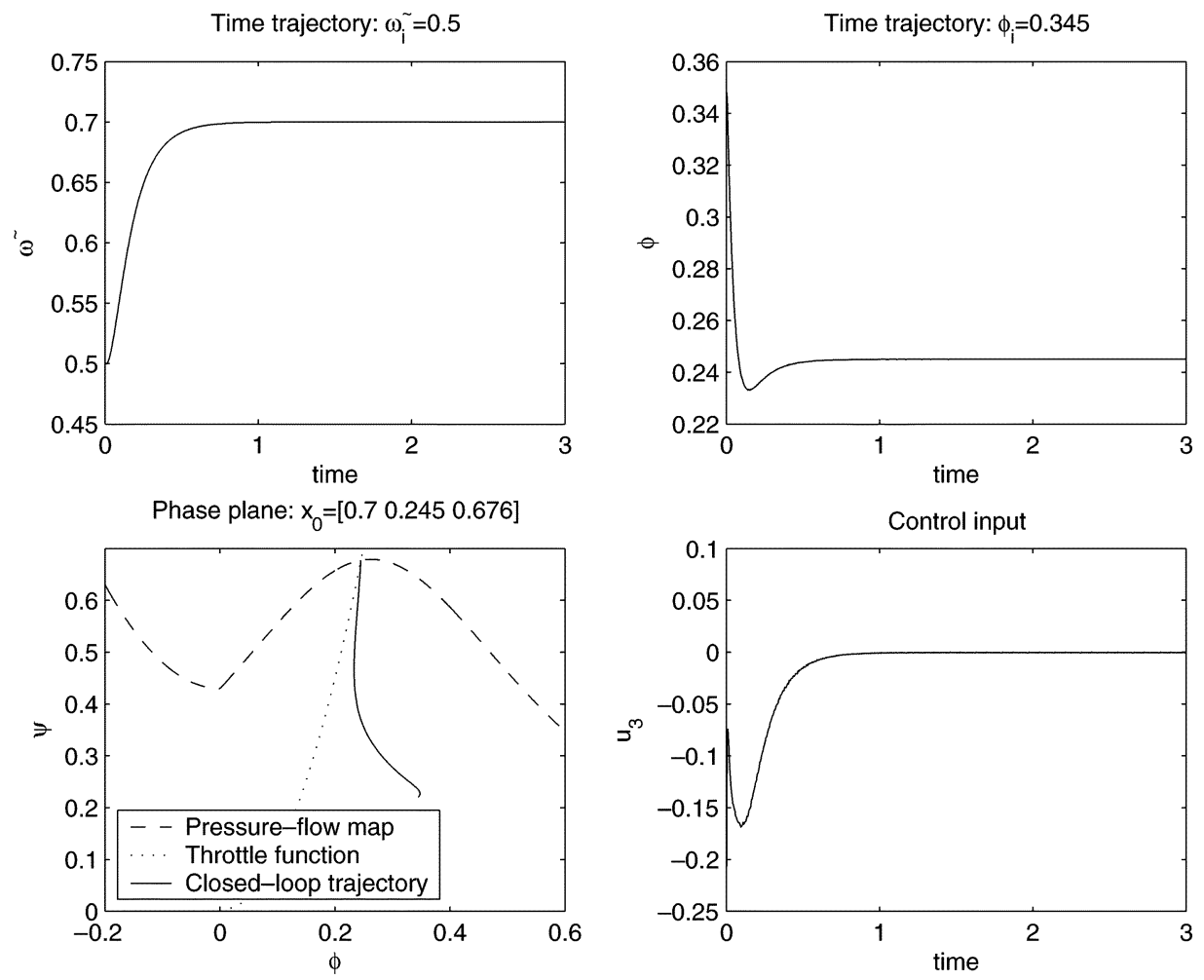

Fig. 3. Time trajectories for system with throttle modulating control.

It is clear to have the bounds for $\Delta f\left(x_{1}, x_{2}\right)$ and $\Delta C_{s s}\left(x_{1}, x_{2}\right)$ as $\delta_{1}=\delta_{2}=0.1$. With the addition of these uncertainties, the transient behavior of the closed-loop system in Case 1 is depicted by the dashed line of Fig. 4, and surge oscillation still exists. When an inner loop control as given in (44) is added on to achieve the global stability of the whole system, as shown

by the dotted line of Fig. 4, the oscillation is found to become smooth with smaller amplitude. However, it does not converge to the equilibrium point. In order to compensate the system uncertainty, as well as inner-loop control, the outer-loop control law in (46)-(47) is employed to drive the oscillating trajectories into the stable equilibrium point. The timing response depicted 


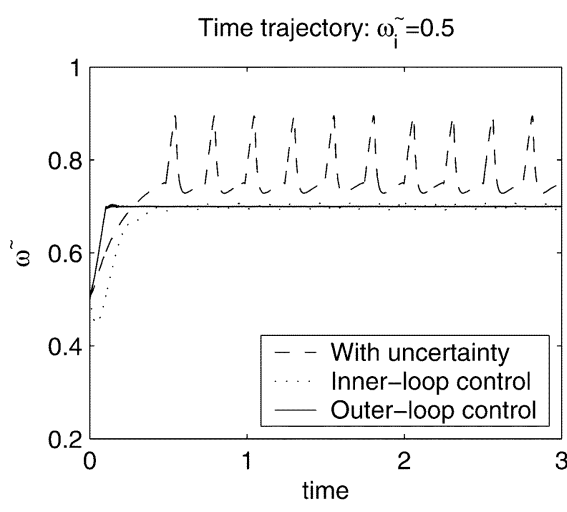

Time trajectory: $\psi_{i}=0.22$

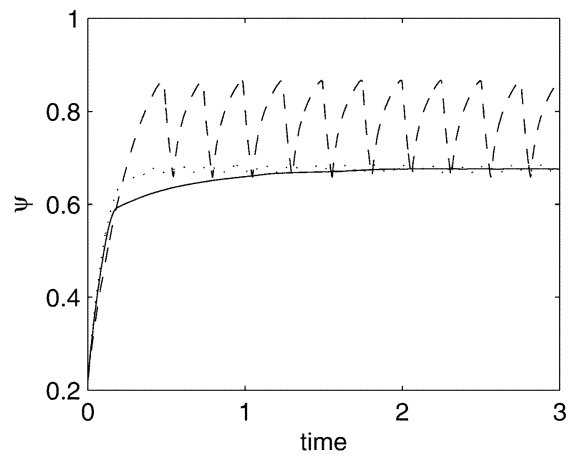

Fig. 4. Time trajectories for system with robust control.

by the solid line of Fig. 4 shows the success of the robust stabilization design.

\section{CONCLUSION}

In this brief, three different control designs are proposed to stabilize the three-state model of a centrifugal compressor with and without uncertainty. When the uncertainty is absent, the global stability of the system can be achieved by a single control input. This design has the same effect as that presented in [3] and [4]. In addition, a two-loop robust control design is attained to achieve system stability with uncertainties appearing in compressor characteristic and spool dynamics. Though the analytical works can guarantee the global stabilization of compression system, the proposed designs depend on the explicit knowledge of system characteristics. To relax the knowledge of system dynamics, instead of global stabilization, a practical stabilization can be achieved by a similar approach.

\section{ACKNOWLEDGMENT}

The authors would like to thank the associate editor and reviewers for their valuable comments and suggestions.

\section{REFERENCES}

[1] B. de Jager, "Rotating stall and surge control: A survey," in Proc. IEEE Conf. Decision Control, New Orleans, LA, 1995, pp. 1857-1862.

[2] G. Gu, S. Banda, and A. Sparks, "An overview of rotating stall and surge control for axial flow compressors," IEEE Trans. Contr. Syst. Technol., vol. 7, pp. 639-647, Nov. 1999.

[3] H. Li, A. Leonessa, and M. Haddad, "Globally stabilizing controllers for a centrifugal compressor model with spool dynamics," in Proc. Amer. Control Conf., 1998, pp. 2160-2164.

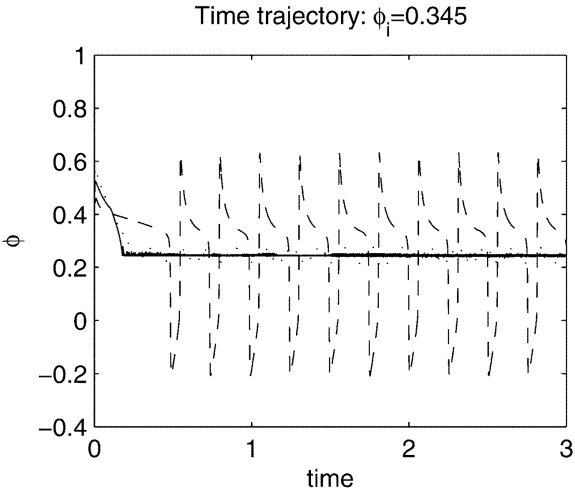

Phase plane: $x_{0}=\left[\begin{array}{lll}0.7 & 0.245 & 0.676\end{array}\right]$

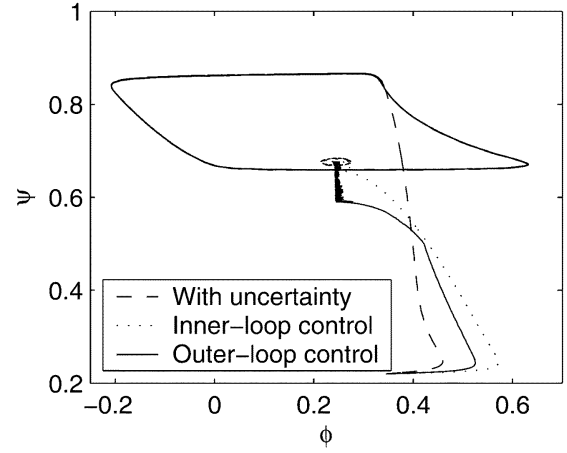

[4] A. Leonessa, M. Haddad, and H. Li, "Globally stabilizing switching controllers for a centrifugal compressor model with spool dynamics," IEEE Trans. Contr. Syst. Technol., vol. 8, pp. 474-482, May 2000.

[5] J. T. Gravdahl and O. Egeland, "Centrifugal compressor surge and speed control," IEEE Trans. Contr. Syst. Technol., vol. 7, pp. 567-579, Sept. 1999.

[6] J. S. Simon and L. Valavani, "A Lyapunov based nonlinear control scheme for stabilizing a basic compression system using a close-coupled control valve," in Proc. Amer. Control Conf., 1991, pp. 2398-2406.

[7] J. T. Gravdahl and O. Egeland, "Compressor surge control using a closecoupled valve and backstepping," in Proc. Amer. Control Conf., 1997, pp. 982-986.

[8] _ "Speed and surge control for a low order centrifugal compressor model," Model Identif. Control, vol. 19, no. 1, pp. 13-29, 1998.

[9] K. E. Hansen, P. Jorgensen, and P. S. Larsen, "Experimental and theoretical study of surge in a small centrifugal compressor," J. Fluid Eng., vol. 103, pp. 391-394, 1981

[10] E. M. Greitzer, "Surge and rotating stall in axial flow compressors: Part 1 and 2," J. Eng. Power, vol. 98, pp. 190-217, 1976.

[11] D.-C. Liaw and E. H. Abed, "Active control of compressor stall inception: A bifurcation-theoretic approach," Automatica, vol. 32, pp. 109-115, 1996.

[12] C. A. Mansoux, D. L. Gysling, and J. D. Paduano, "Distributed nonlinear modeling and stability analysis of axial compressor stall and surge," in Proc. Amer. Control Conf., 1994, pp. 2305-2316.

[13] M. Krstić, I. Kanellakopoulos, and P. V. Kokotović, Nonlinear and Adap tive Control Design. New York: Wiley, 1995.

[14] M. Krstić, J. M. Protz, J. D. Paduano, and P. V. Kokotović, "Backstepping designs for jet engine stall and surge control," in Proc. IEEE Conf. Decision Control, New Oleans, LA, 1995, pp. 3049-3055.

[15] A. Leonessa, V. Chellaboina, and W. M. Haddad, "Robust stabilization of axial flow compressors with uncertain pressure-flow maps," IEEE Trans. Contr. Syst. Technol., vol. 8, pp. 466-473, May 2000.

[16] D.-C. Liaw and J.-T. Huang, "Robust stabilization of axial flow compressor dynamics via sliding mode design," ASME J. Dynam. Syst. Meas. Control, vol. 123, pp. 488-495, 2001

[17] D. A. Fink, N. A. Cumpsty, and E. M. Greitzer, "Surge dynamics in a free-spool centrifugal compressor system," J. Turbomachinery, vol. 114, pp. 321-332, 1992.

[18] J. T. Gravdahl, O. Egeland, and S. O. Vatland, "Drive torque actuation in active surge control of centrifugal compressors," Automatica, vol. 38, no. 11, pp. 1881-1893, 2002. 